\title{
G s.mextem \\ Diffuse sclerosing variant of papillary thyroid carcinoma: a case report
}

\section{Siyao Wang ( $\triangle$ m13704931681@163.com )}

China Medical University

\section{Rui Wang}

the First Affiliated HospitaDepartment of Pathology, the First Affiliated Hospital and College of Basic Medical Sciences of China Medical Universityl and Coll

\section{Ruize Sun}

Department of Pathology, the First Affiliated Hospital and College of Basic Medical Sciences of China Medical University

\section{Xiaoman Luo}

Department of Pharmaceutical Biotechnology, Queen's University of Belfast Joint College of China Medical University

\section{Yue Xuan}

Department of Pharmaceutical Biotechnology, Queen's University of Belfast Joint College of China Medical University

\section{Xiaohan Sun}

Department of Pharmaceutical Biotechnology, Queen's University of Belfast Joint College of China Medical University

\section{Aoxue Li}

Department of Pharmaceutical Biotechnology, Queen's University of Belfast Joint College of China Medical University

\section{Yilin Cong}

Department of Pharmaceutical Biotechnology, Queen's University of Belfast Joint College of China Medical University

\section{Jiayan Zhang}

Department of Pharmaceutical Biotechnology, Queen's University of Belfast Joint College of China Medical University

\section{Pengcheng Li}

Department of Pathology, the First Affiliated Hospital and College of Basic Medical Sciences of China Medical University

\section{Yuan Luo}

Department of Pathology, the First Affiliated Hospital and College of Basic Medical Sciences of China Medical University 
Department of Pathology, the First Affiliated Hospital and College of Basic Medical Sciences of China Medical University

\section{Yang Liu}

Department of Pathology, the First Affiliated Hospital and College of Basic Medical Sciences of China Medical University

\section{Case Report}

Keywords: Case report, papillary thyroid carcinoma, Hashimoto background, psammoma bodies

Posted Date: March 10th, 2020

DOl: https://doi.org/10.21203/rs.3.rs-16557/v1

License: (9) This work is licensed under a Creative Commons Attribution 4.0 International License. Read Full License 
Siyao Wang ${ }^{1}$, Rui Wang ${ }^{1}$, Ruize Sun ${ }^{1}$, Xiaoman Luo ${ }^{2}$, Yue Xuan ${ }^{2}$, Xiaohan Sun ${ }^{2}$, Aoxue Li ${ }^{2}$, Yilin Cong ${ }^{2}$,

${ }^{1}$ Department of Pathology, the First Affiliated Hospital and College of Basic Medical Sciences of

5 China Medical University, Shenyang, 110122, PR China

*Corresponding author: Tel.: +86 24 23261638; fax: +86 2423261638 .

7 E-mail address: liuyanglovebee@163.com

8

${ }^{2}$ Department of Pharmaceutical Biotechnology, Queen's University of Belfast Joint College of China Medical University

Abstract

Background: The diffuse sclerosing variant of papillary thyroid carcinoma (DSVPC) is a rare variant of papillary thyroid carcinoma with features of strong ability of invasion, metastasis, relapse, and mortality. Its diagnosis is difficult to obtain because of the special differentiation state of the cancer cells.

Case Presentation: A 21-year-old woman arrived at the First Affiliated Hospital of China Medical University in October 2019 because of a peach-pit-sized mass that she had noticed 1 month before. Color duplex ultrasonography findings suggested a thyroid nodule.

Conclusions: Based on findings of the specialist examination, physical examination, and thyroid ultrasonography, the patient was initially diagnosed with thyroid neoplasm. Under general 
anesthesia, thyroidectomy was performed, removing the right lobe and isthmus, and biopsy specimens were obtained from lymph node groups III, IV, and VI on the right side. The final histological diagnosis was DSVPC.

Keywords: Case report; papillary thyroid carcinoma; Hashimoto background; psammoma bodies.

\section{Background}

Papillary thyroid cancer (PTC) is a common malignant tumor of the thyroid, accounting for $75 \%-$ $85 \%$ of all cases of thyroid tumors. It manifests as a mass of irregular and unclear boundary with a single solid hypoechoic nodule and scattered granular or dense sandy calcification. Blood supply in the nodule is abundant, with high blood flow speed and resistance. Based on the tumour size, boundary type, architecture, cellular characteristics, additional components, and stromal features, PTC can be classified into diffuse sclerosing and Warthin-like variants [1-2].

The diffuse sclerosing variant of papillary thyroid carcinoma (DSVPC) was first described by Vickeryin in 1985 [3]. It is an uncommon subtype of PTC with a high risk of recurrence, accounting for $0.7 \%-6.6 \%$ of all cases of PTC. Higher prevalence was noted in women around the age of 20 years and the pediatric population. Its typical histopathologic characteristics include extensive lymph vascular invasion, dense lymphocytic infiltration, and presence of psammoma bodies. Ultrasonographic findings include diffuse enlargement of the thyroid and humus punctate 
calcification. DSVPC usually involves the unilateral or bilateral thyroid diffusely. It is characterized by strong ability of invasion, metastasis, relapse, and mortality [4-6] but shows good outcomes. However, compared to classical PTC, the recurrence rate of DSVPC is higher [7-8].

\section{Case presentation}

A 21-year-old woman arrived at our hospital because of a peach-pit-sized mass that she had noticed one month before. Color duplex ultrasonography findings suggested a thyroid nodule. The patient did not complain of pain, dysphagia, choking, or hoarseness. She had no history of fever, weight loss, palpitation, dyspnea, or hypermetabolic symptoms, such as polyphagia, emaciation, or rage. She had no disturbances in appetite, sleeping, or excretion. She had no history of tuberculosis, hepatitis, allergy, hypertension, coronary heart disease., diabetes, blood transfusion, trauma, or surgery.

Specialist examination showed that the neck was symmetrical, and the trachea was in the middle.

An abnormal nodule was detected on the right side of the neck with a diameter of approximately $3 \mathrm{~cm}$, and no tubercles could be palpated on the left side of the neck. The surface of the nodule was smooth, without compression, and moved up and down upon swallowing. No clearly enlarged lymph nodes could be palpated on the neck. Physical examination revealed a body temperature of $36.5^{\circ} \mathrm{C}$, pulse rate of 74 beats $/ \mathrm{min}$, respiratory rate of 14 breaths $/ \mathrm{min}$, and blood pressure of $113 / 74 \mathrm{mmHg}$. Auscultation revealed no abnormal noise in the cardiopulmonary valve area. The 
liver and spleen were not enlarged. Thyroid ultrasound showed changes in thyroid echogenicity. The hypoechoic (decreased echogenicity compared to the adjacent thyroid parenchyma) and diffusely punctate calcification (Thyroid Imaging Reporting and Data System-5) in the right lobe of the thyroid were consistent with Hashimoto's disease. Lymph node groups III, IV, and VI on the right side of the neck were enlarged with calcification, and secondary lymph nodes on the left side of the neck were enlarged. No significant abnormalities were detected in the ultrasonic structure. Based on the onset of the neck mass, cosmetic appearance, and results of the specialist examination, physical examination, and thyroid ultrasonography, the patient was initially diagnosed with thyroid neoplasm, which is different from thyroglossal tract cysts. After admission, all examinations were completed. Under general anesthesia, thyroidectomy was performed, removing the right lobe and isthmus, and biopsy specimens were obtained from lymph node groups III, IV, and VI on the right side. Histopathological sections showed nodular goiter with Hashimoto's disease in the right lobe of the thyroid. Epithelial dysplasia and scattered psammoma bodies were found in some areas. Hyperplasia was observed in the lymph node group III area on the right side. Some psammoma bodies were scattered in the envelope of one group IV lymph node on the right side, consistent with lymph node metastasis. Metastasis was also found in group IV lymph nodes on the right side. Molecular pathological results indicated no metastasis in the anterior laryngeal lymph nodes. Appropriate treatment was provided postoperatively. The patient recovered well with good healing of the neck incision and had no hoarseness, cough when drinking water, numbness of the mouth or lips, convulsions of the hands or feet, or dyspnea. The drainage tube was pulled out, and the patient was discharged with the approval of a senior physician. At the time of discharge, the patient was cured. 


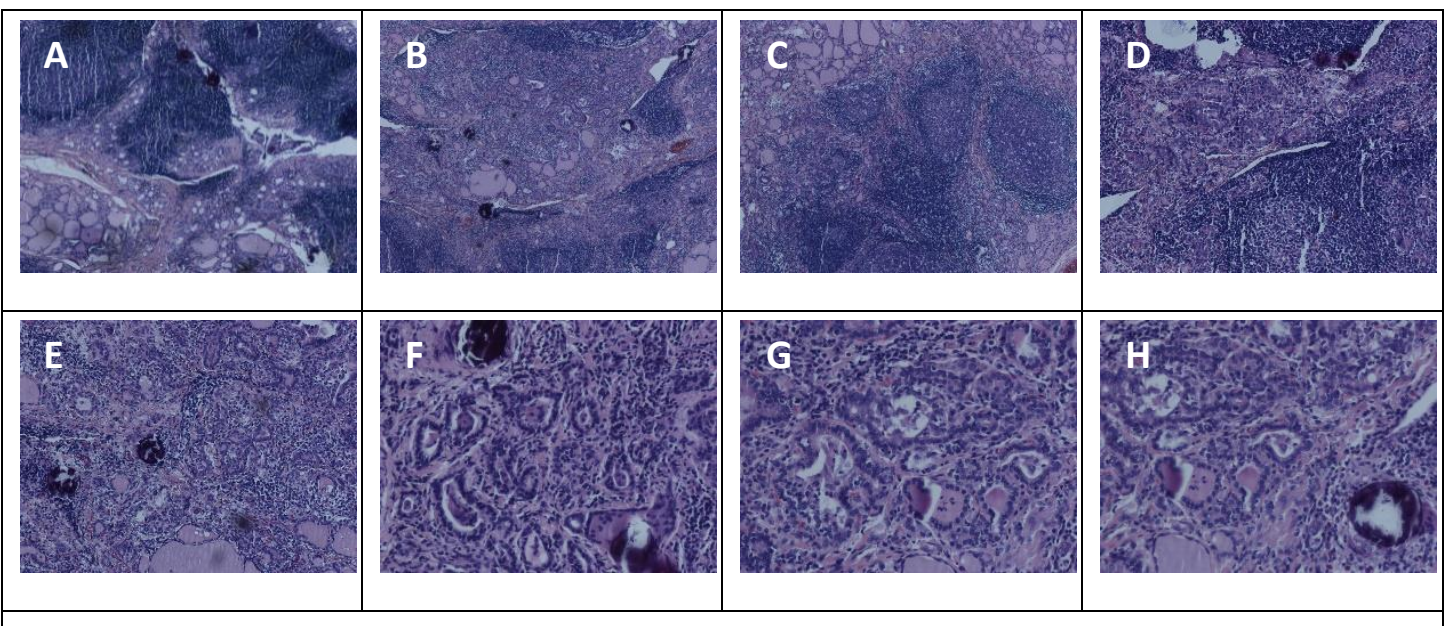

Figure 1. (A-E) Hashimoto's disease is found between the thyroid follicles. Primary thyroid carcinoma is diagnosed on hematoxylin \& eosin (H\&E) staining (ob. $4 \times$ ). (F-H) In the background of Hashimoto's disease, primary cancer nests are seen around the psammoma bodies (H\&E staining; ob. $10 \times$ ).

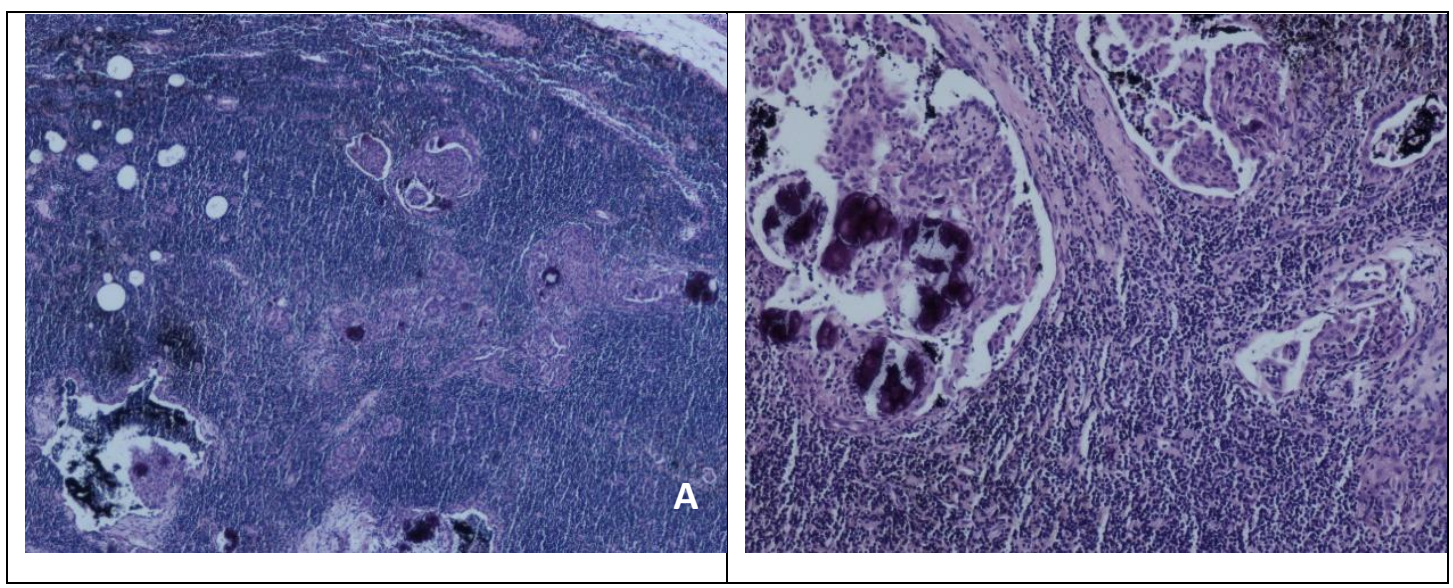




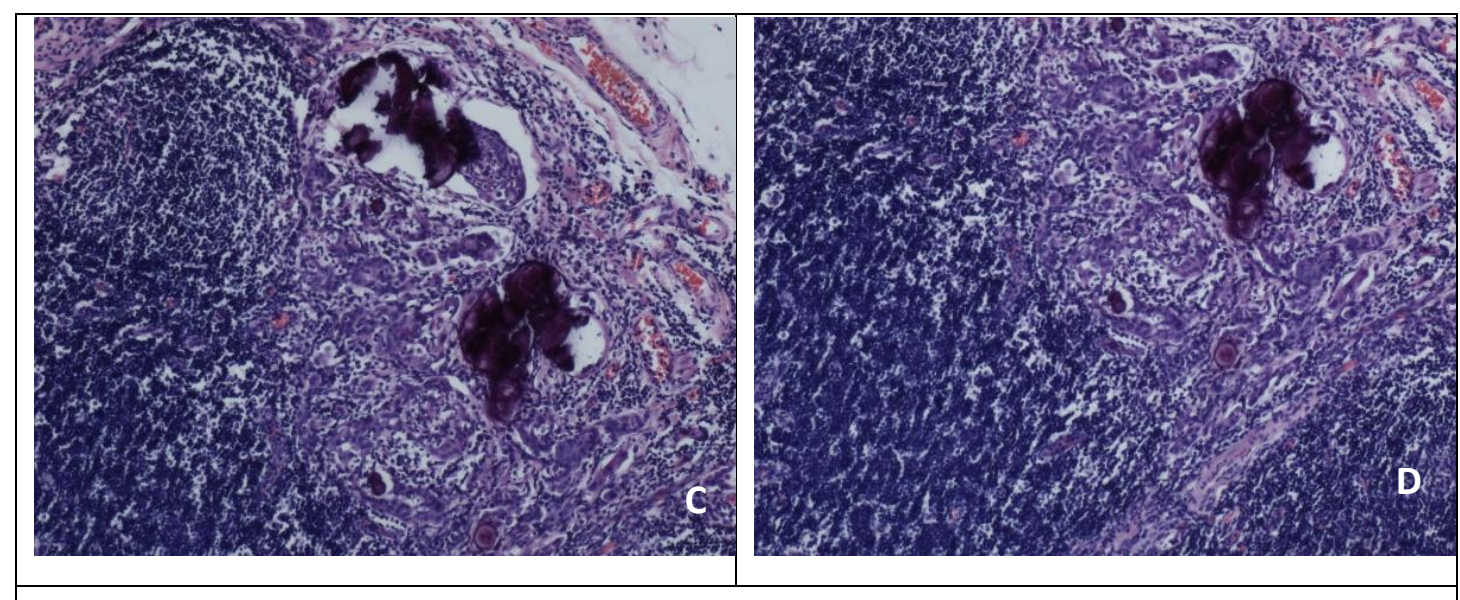

Figure 2. (A) Metastatic cancer cell aggregates are present in the lymphatic spaces (hematoxylin \& eosin [H\&E] staining, ob. $4 \times$ ) (B-D) Some calcified psammoma bodies are found in the background of Hashimoto's disease, and tumor cells aggregates are seen around the psammoma bodies. The pathological diagnosis is the diffuse sclerosing variant of papillary carcinoma of the thyroid (H\&E staining, ob. $10 \times$ )

84

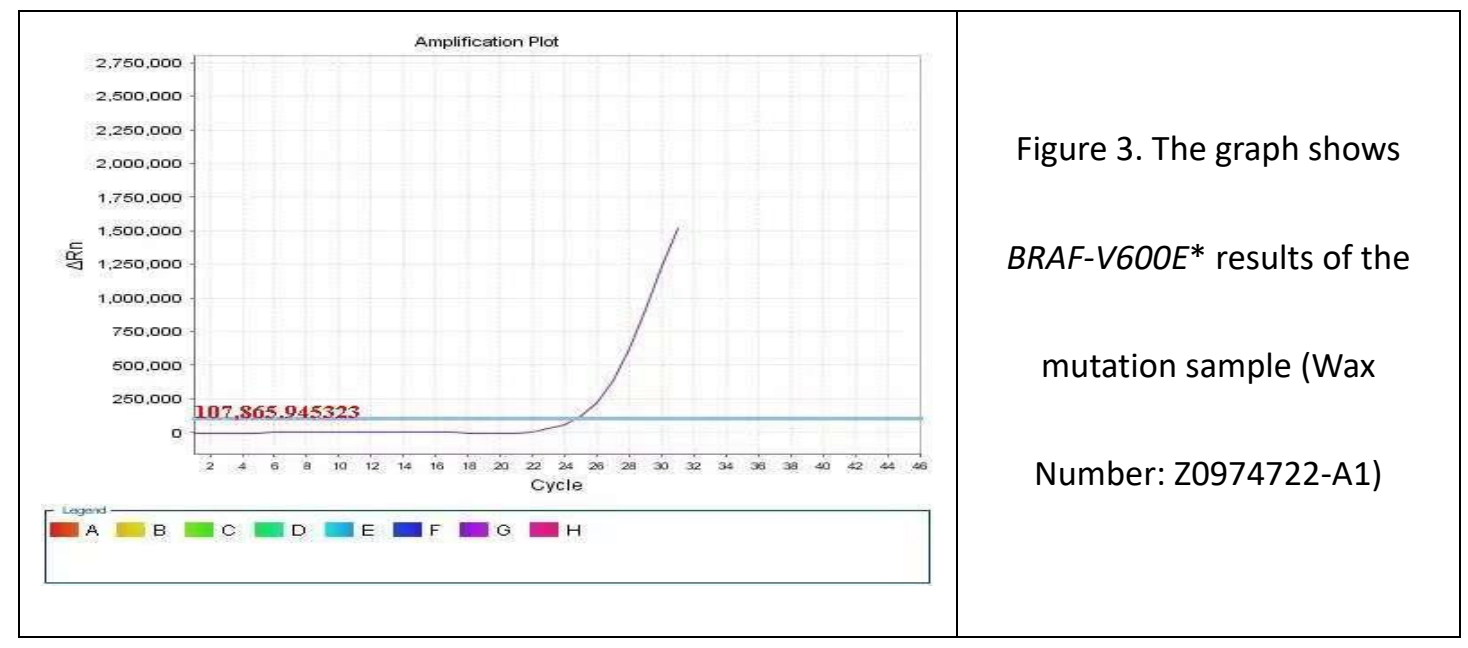

85 
DSVPC is a rare variant of thyroid cancer that mainly affects women around 20 years of age. The RET oncogene in PTC shows a high correlation with the occurrence of DSVPC, which could explain the higher prevalence of DSVPC in the younger population [9-10]. Furthermore, in a series, Spinelli et al. reported that DSVPC frequently develops in the background of Hashimoto's thyroiditis and positive antithyroid antibodies [8]. DSVPC has the characteristics of thyroid cancer as well as excessive fibrosis, presence of psammoma bodies, frequent squamous metaplasia, and abundant lymphocytic infiltration [11-13]. A meta-analysis revealed that DSVPC is more aggressive and has higher incidences of tumor invasion, metastasis, recurrence, and mortality compared to classical PTC [4].

Mutations in the BRAF gene are common in human cancers, particularly thyroid cancer. According to previous reports, BRAFV-600E mutates frequently in classic PTC but rarely in DSVPC [14-15], contrary to cases reported in Korea. In a previous study, $61 \%$ of 98 patients with DSVPC had the BRAF-V600E mutation [16]. Therefore, the association between DSVPC and BRAF-V600E mutation is unclear. Moreover, some studies indicated that the BRAF mutation is associated with a high mortality and poor prognosis [16-17].

Due to the rarity of DSVPC, the diagnosis is often incorrect or missed by both clinicians and pathologists. Therefore, pathological examination is the most important method for the diagnosis of DSVPC. Moreover, if Hashimoto's thyroiditis is present with diffuse involvement of the thyroid gland, the patient might be clinically diagnosed with Hashimoto's thyroiditis, leading to delayed 
treatment [18-20]. Among the present cases, patients with DSVPC are most frequently diagnosed with Hashimoto's thyroiditis, and a case reports shows that DSVPC can mimic benign Riedel's thyroiditis [21]. The main issue is the appearance of the fibrotic thyroid gland. During the process, fibrosis spreads to the adjacent neck structures and extends beyond the thyroid envelope, which helps differentiate it from Hashimoto's thyroiditis. Patients with Riedel's thyroiditis always have a hard and "woody" painless mass in the front of the neck. According to a case study in 2005, DSVPC might be masked by florid lymphocytic thyroiditis in preoperative fine-needle aspiration cytology [18]. Moreover, a rare case of DSVPC that arose from underlying Hashimoto's thyroiditis was reported in 2016 in a 22-year-old woman, and ultrasonography showed innumerable diffuse microcalcifications instead of a typical malignant-appearing nodule [22].

In addition, ultrasonography of the cervical lymph nodes can be used as an effective auxiliary examination. Ultrasonography in our case revealed similar features as previously reported: abnormal thyroid echogenicity, hypoechoic areas, diffuse calcification, and lymph node enlargement, consistent with Hashimoto's disease of the thyroid. Most patients with DSVPC have lymph node metastasis at an early stage $[4,23]$. Therefore, cervical metastatic lymph nodes on ultrasonography are an indirect sign of DSVPC and can be a clue for the correct diagnosis. Furthermore, because of the higher rate of extrathyroidal extension and lymph node and distant metastases, which are more obvious in the advanced stage of DSVPC, the disease might be more easily diagnosed in the advanced stage [24]. However, particularly in cases without nodular masses, the diffuse calcification on ultrasonography often mimics chronic thyroiditis, which is another 
130

131

132

Ethics approval and consent to participate: Not applicable. and a more positive outcome can be obtained.

List of abbreviations

DSVPC: Diffuse sclerosing variant of papillary thyroid carcinoma

PTC: Papillary thyroid cancer

Declarations

reason for delayed treatment [19]. DSVPC is an aggressive thyroid cancer, but early diagnosis and appropriate treatment may show good outcomes $[6,25]$. As the early diagnosis and treatment can lead to a good prognosis, a high index of suspicion must be kept to avoid misdiagnosis.

In conclusion, the ultrasonographic finding of diffusely scattered microcalcifications, particularly accompanied by cervical lymph node metastasis, should raise the suspicion for DSVPC. When DSVPC is suspected based on ultrasonographic findings, clinicopathological examination of the thyroid should be performed for the definitive diagnosis. Pathological examination demonstrating the cancer cells around abundant psammoma bodies in a background of Hashimoto's thyroiditis is highly suggestive of DSVPC. Using these features, DSVPC can be diagnosed easier in the early stage,

Consent for publication: We obtained informed consent from the patient for the publication of this 
case report.

150 Availability of data and materials: Not applicable

151 Competing interests: The authors declare that they have no competing interests.

152 Funding: The authors have no funding to disclose.

153 Authors' contributions: SYW collect the clinical data and writing the paper. RW, RZS and YL provided

154 the staining images and clinical information

155 Acknowledgements: Not applicable 


\section{Figures}

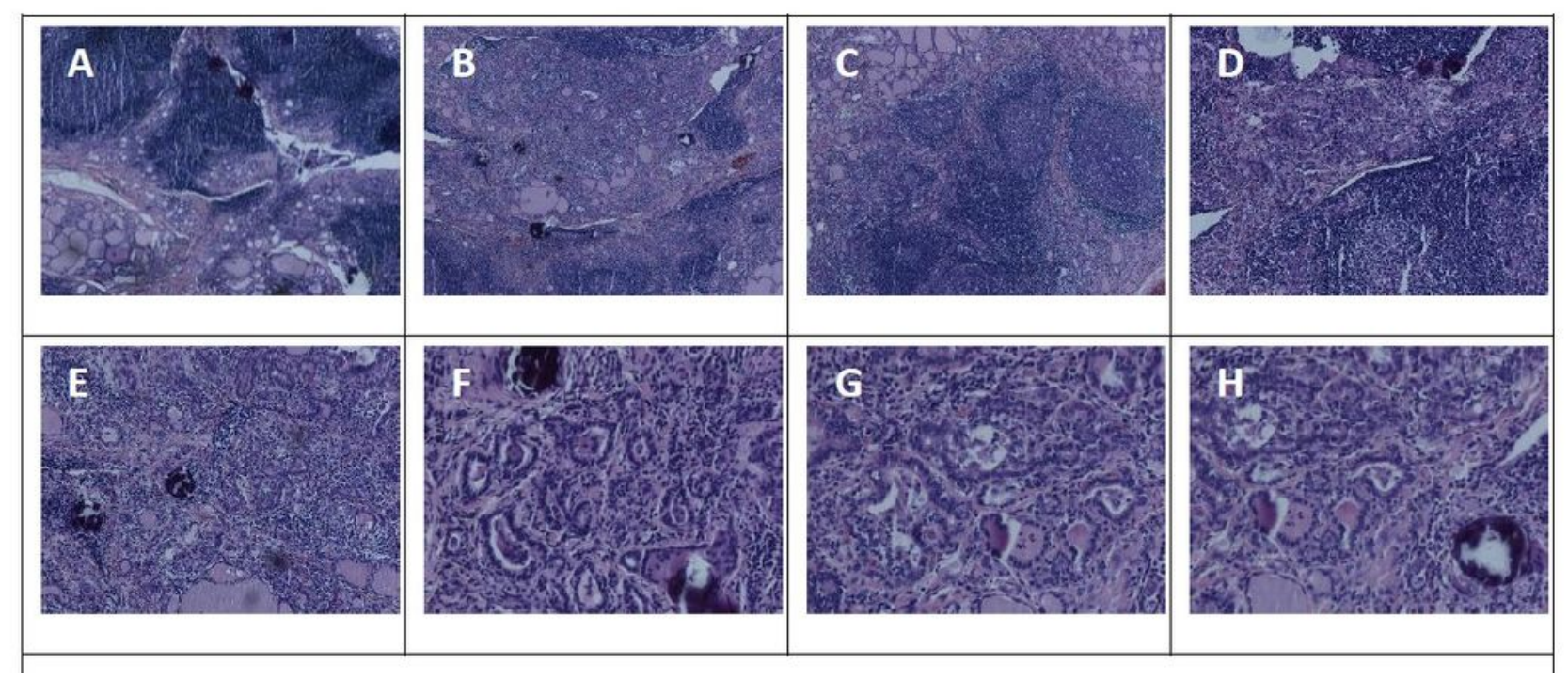

Figure 1

(A-E) Hashimoto's disease is found between the thyroid follicles. Primary thyroid carcinoma is diagnosed on hematoxylin \& eosin (H\&E) staining (ob. $4 \times x)$. $(\mathrm{F}-\mathrm{H})$ In the background of Hashimoto's disease, primary cancer nests are seen around the psammoma bodies (H\&E staining; ob. 10xx).

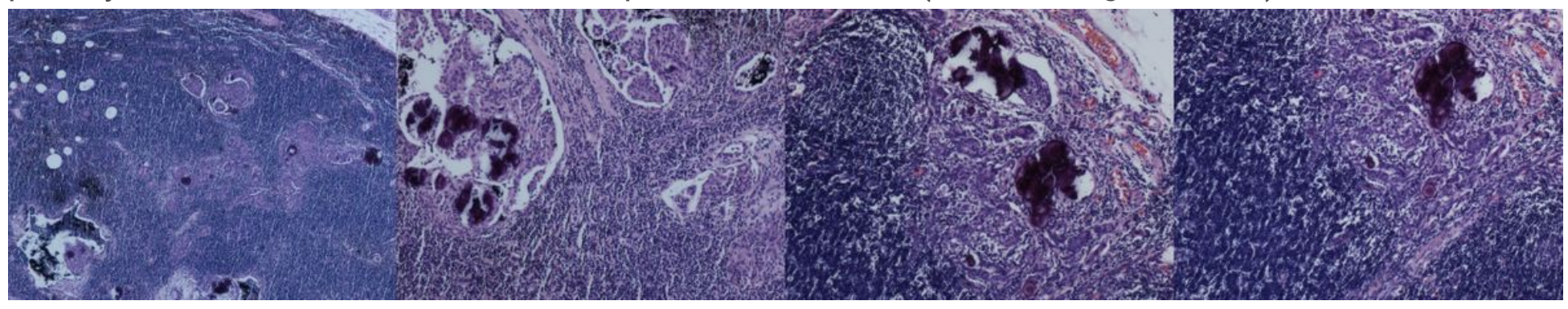

\section{Figure 2}

(A) Metastatic cancer cell aggregates are present in the lymphatic spaces (hematoxylin \& eosin [H\&E] staining, ob. $4 \times x)(B-D)$ Some calcified psammoma bodies are found in the background of Hashimoto's disease, and tumor cells aggregates are seen around the psammoma bodies. The pathological diagnosis is the diffuse sclerosing variant of papillary carcinoma of the thyroid (H\&E staining, ob. 10xx) 


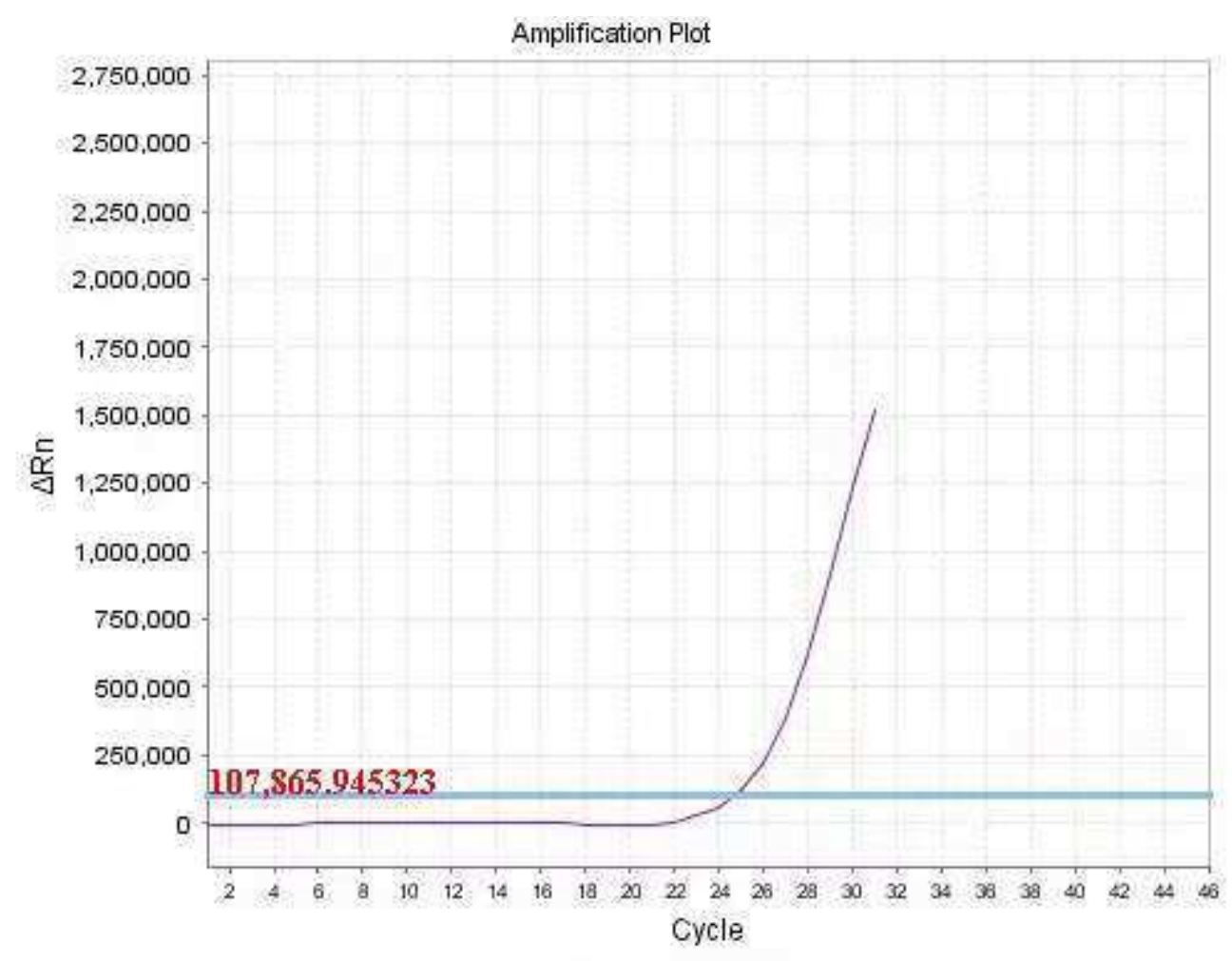

$A \square B \square C \square D \square E \square F \square G \square H$

Figure 3

The graph shows BRAF-V600E* results of the mutation sample (Wax Number: Z0974722-A1)

\section{Supplementary Files}

This is a list of supplementary files associated with this preprint. Click to download.

- CAREchecklist.pdf 